\title{
CARACTERIZACIÓN FÍSICO-QUÍMICA DEL HUEVO DEL CAIMÁN LLANERO, CROCODYLUS INTERMEDIUS, GRAVES 1819
}

\author{
Julián Morales-Arango, Marcela Duarte-Guerrero \& Herly ZúÑIGa \\ Universidad de Cundinamarca, Diagonal 18 No. 20-29 Fusagasugá, \\ Cundinamarca, COLOMBIA. \\ jandresmoran@gmail.com,marchdu1@gmail.com, herlyzuga@hotmail.com
}

\begin{abstract}
RESUMEN
Se determinaron algunas características físico-químicas del huevo de Crocodylus intermedius tales como peso, morfometría, gravedad específica, grosor de la cáscara, calidad y cantidad del albumen denso, concentración de calcio y fósforo inorgánico de la cáscara, porcentaje de humedad en vitelo y albumen, contenido de proteína y contenido de grasa cruda. Los resultados obtenidos fueron: peso (121.33 g), diámetro mayor longitudinal $(84.12 \mathrm{~mm})$, diámetro mayor transversal $(51.64 \mathrm{~mm})$, longitud perimetral (228.5 mm), ancho perimetral $(173.17 \mathrm{~mm})$, gravedad específica (1.11-1.14), grosor de la cáscara del huevo en el polo ancho $(296.91 \mu \mathrm{m})$, grosor de la cáscara en el polo angosto $(275.91 \mu \mathrm{m})$, grosor de la cáscara en la zona ecuatorial (313.75 $\mu \mathrm{m})$, cantidad y calidad de albumen denso (73.7 unidades Haugh), concentración de calcio inorgánico (43\%), concentración de fósforo inorgánico (1.84\%), porcentaje de humedad en vitelo (56.48\%), porcentaje de humedad en albumen $(91.24 \%)$, contenido de proteína cruda en vitelo (18.54\%), contenido de proteína cruda en albumen (6.68\%), contenido de grasa cruda en vitelo (19.30\%). Los resultados obtenidos de los análisis físico-químicos en los huevos de C. intermedius son similares a los publicados por otros autores en diferentes especies de crocodilianos.

Palabras Clave: caracterización físico-química, Crocodylus intermedius, huevos.
\end{abstract}

\begin{abstract}
Some physicochemical characteristics of Crocodylus intermedius eggs were determined such as weight, morphometrics, specific gravity, shell thickness, quality and quantity of dense albumen, concentration of calcium and inorganic shell phosphorus, percentage of moisture in yolk and albumen, protein content and raw fat content. The results obtained were: weight (121.33 g), longitudinal longer diameter $(84.12 \mathrm{~mm})$, transversal longer diameter $(51.64 \mathrm{~mm})$, perimetral length $(228.5 \mathrm{~mm})$, perimetral width $(173.17 \mathrm{~mm})$, specific gravity (1.115-1.14), shell thickness in the wide pole $(296.91 \mu \mathrm{m})$, shell thickness in the narrow pole $(275.91 \mu \mathrm{m})$, shell thickness in the equatorial area $(313.75 \mu \mathrm{m})$, quantity and quality of dense albumen (73.7 Haugh units), concentration of inorganic calcium (43\%), concentration of inorganic phosphorus $(1.84 \%)$, percentage of humidity in albumen (91.24\%), content of raw protein in yolk (18.54\%), content of raw protein in albumen $(6.68 \%)$, content of raw fat in yolk $(19.30 \%)$. The results of the physico-chemical analyses of $C$. intermedius eggs are similar to those of different crocodilian species reported elsewhere.
\end{abstract}

Key Words: physicochemical characterization, Crocodylus intermedius, eggs. 


\section{INTRODUCCIÓN}

Una de las especies de crocodilianos menos conocidas de Sur América es el cocodrilo del Orinoco (Crocodylus intermedius) restringida a las orillas de la cuenca del bajo y medio Orinoco de Colombia y Venezuela (Thorbjarnarson \& Hernández 1993). Es uno de los cocodrilos que alcanza mayores tallas, registrándose ejemplares cercanos a los $7 \mathrm{~m}$ de longitud total (Rodríguez \& Ramírez 2002).

Esta especie se encuentra catalogada en peligro crítico (CR, criterios Alc, C2a) por la UICN (2001) y por los libros rojos de Colombia (criterio C2a) (Rodríguez \& Ramírez 2002). El caimán llanero es la segunda especie de herpetofauna más amenazada de Colombia. Esta especie fue catalogada por la Comisión de Supervivencia de Especies de la UICN como una de las doce especies animales más amenazadas del planeta y de la cual estimó la existencia de no más de 1500 individuos en el medio natural. Está catalogada por el CITES en el apéndice I (Rueda 1999). Adicionalmente, C. intermedius se encuentra protegido por la resolución 411 de 1968 expedida por el Ministerio de Agricultura de Colombia la cual sancionó indefinidamente su caza. Con base en los estudios de poblaciones realizadas entre 1994 y 1997, el Ministerio del Medio Ambiente de Colombia expidió la resolución 0676 por la cual se declara a la especie en peligro de extinción y se ordena la estructuración de un Plan Nacional para su recuperación y conservación (Rodríguez \& Ramírez 2002).

Los Crocodylia han desarrollado un ciclo reproductor estrechamente sincronizado con los cambios estacionales del ambiente en que viven (Lance 1986). En $C$. intermedius la época de celo se presenta en los meses de septiembre y octubre (Medem 1981). La nidificación ocurre al comienzo de la estación seca y los huevos eclosionan cuando el nivel del agua comienza a subir a principio de la estación lluviosa. Las hembras nidifican en los mismos sitios año tras año (Thorbjarnarson \& Hernández 1993). La biología básica reproductiva y la embriología entre crocodilianos es similar entre todas las especies, el periodo de incubación se divide en dos etapas, la primera es el desarrollo embrionario u organogénesis, caracterizado por la diferenciación de estructuras morfológicas, y la segunda es la etapa fetal en la que ocurre crecimiento somático (Ardila et al. 1999).

Los huevos de aves, quelonios y crocodilianos contienen una gran cantidad de vitelo, son huevos de tipo telolecítico y en relación con la medida del huevo hay un pequeño embrión al momento de la postura (Manolis et al. 1986). Los embriones de estos tres grupos desarrollan las mismas membranas extraembrionarias (amnios, corion y alantoides) las cuales dividen al huevo en compartimientos similares. El huevo contiene suplementos con la mayoría de los nutrientes para el desarrollo embrionario, mientras el medio ambiente fija la temperatura de incubación y suministra un medio para el intercambio de gases y agua. 
Todos los crocodilianos ponen huevos elípticos rígidos, de cáscara calcárea. La cáscara está formada por dos membranas fibrosas sobre la cual hay una capa relativamente gruesa de carbonato de calcio. La capa calcárea está compuesta de cristales de calcio, la capa cristalina ocasionalmente es interrumpida por poros que facilitan el intercambio de agua y gases respiratorios entre el embrión y el ambiente del nido. Estos poros tienden a ser más numerosos en el ecuador del huevo que en cualquiera de los polos (Gans \& Huey 1982).

Este estudio tuvo como objetivo determinar las siguientes características del huevo de $C$. intermedius: peso, morfometría, gravedad específica, grosor, color, resistencia a la fracturabilidad de la cáscara, calidad y cantidad del albumen denso, concentración de calcio y fósforo inorgánico, porcentaje de humedad en vitelo y albumen, contenido de proteína y contenido de grasa cruda, y comparar estas características con las de los huevos de otros crocodrilianos.

\section{MATERIAL Y MÉTODOS}

Se utilizaron 12 huevos de C. intermedius, donados por la Estación de Biología Tropical Roberto Franco de la Universidad Nacional de Colombia-(Villavicencio, Meta) y obtenidos de animales criados en cautiverio manejados bajo condiciones homogéneas y controladas. Estos huevos eran infértiles ya que no mostraron desarrollo de la banda opaca dentro las 24 horas siguientes a la postura, ni fue posible detectar fluido subembrionario o un disco embrionario (Grahame \& Manolis 1986).

\section{Determinación de variables físicas}

Los huevos se pesaron individualmente de acuerdo con Ramírez (1999) en una balanza electromecánica Sartorius con precisión de 0.0001 g. El peso del vitelo, albumen y cáscara se determinó en una balanza analítica Sartorius con precisión de $0.0001 \mathrm{~g}$ y se determinó el porcentaje de participación de cada uno de estos componentes con respecto al peso del huevo.

Las medidas del huevo se tomaron con un calibrador con precisión de $0.05 \mathrm{~mm}$ y una cinta plástica milimetrada. Se realizaron mediciones sobre el diámetro mayor longitudinal, el diámetro mayor transversal, la longitud perimetral y el ancho perimetral de los huevos (Ramírez 1999).

El índice de forma del huevo (relación porcentual entre el diámetro máximo y la longitud máxima del huevo) se definió mediante la fórmula:

$$
\mathrm{IF}=\frac{\text { ancho }}{\text { largo }} * 100
$$


Un índice inferior a 72 indica que se trata de un huevo excesivamente largo y un índice superior a 76 indica que se trata de un huevo redondo (González 1986).

Se utilizó la metodología propuesta por Ramírez (1999) para determinar la flotabilidad del huevo en diferentes soluciones salinas. La gravedad específica de cada solución se calculó mediante la fórmula:

$$
\mathrm{GE}=\frac{\mathrm{W}_{3}-\mathrm{W}_{1}}{\mathrm{~W}_{2}-\mathrm{W}_{1}}
$$

donde $\mathrm{W}_{1}$ corresponde al peso del picnómetro vacío y seco, $\mathrm{W}_{2}$ al peso del picnómetro con agua y $\mathrm{W}_{3}$ al peso del picnómetro con solución salina.

El grosor de la cáscara se midió con un micrómetro Moore and Wright con precisión de $0.01 \mathrm{~mm}$. Las lecturas se efectuaron sobre el polo ancho, polo angosto y zona ecuatorial de cada huevo.

La fracturabilidad de la cáscara se determinó en un texturómetro Shimadzu modelo $\mathrm{E}_{2}$ Test. Se realizaron cinco repeticiones sobre cada huevo tomando muestras al azar de aproximadamente $1 \mathrm{~cm}^{2}$ de cada polo.

El color de la cáscara se determinó en un colorímetro de sólidos (ColorTec PCM/PSM color meter) utilizando el sistema desarrollado por la Comisión Internacional de Luminosidad (CIE 1986).

La calidad del albumen denso se valoró de acuerdo con los procedimientos del Instituto Mexicano de Normalización y Certificación (2004).

\section{Determinación de variables químicas}

La concentración de fósforo inorgánico en la cáscara se evaluó por el método Vanadato-Molibdato, basado en la metodología propuesta por la Universidad de Oviedo (2003) con modificaciones que consistieron en la disolución de $1 \mathrm{~g}$ de cáscara con $8 \mathrm{ml}$ de $\mathrm{HCl} 0.1$ y $1 \mathrm{ml}$ de $\mathrm{HNO}_{3}$ concentrado, procediendo a realizar ebullición suave por 10 min hasta la aparición de humos blancos y continuando con la metodología propuesta.

La concentración de calcio inorgánico en la cáscara se determinó por el método de valoración complexométrica según la Norma Técnica Española UNE 77040 del 2002, con la modificación de reemplazar el calcón carboxílico por murexida + sulfato de potasio.

La humedad se determinó en vitelo y albumen según la metodología de la A.O.A.C (1984).

La proteína cruda en vitelo se determinó por dos métodos buscando el más adecuado para este tipo de muestra. Los métodos empleados fueron el método Kjeldahl con el equipo Buchi B316 y el método Biuret-Lowry.

La medición de grasa total se realizó por medio del método Soxhlet en un equipo Buchi system B811. 


\section{Análisis estadístico}

Se probó la normalidad de las variables de los huevos y se aplicó estadística paramétrica. Se calculó la media y la desviación estándar del peso, variables morfométricas, grosor de la cáscara, gravedad específica, humedad, calidad del albumen denso, calcio y fósforo inorgánico en la cáscara, proteína y grasa total usando las funciones estadísticas para poblaciones de EXCEL Microsoft ${ }^{\circledR}$ Office XP.

Se realizaron análisis de varianza para establecer posibles diferencias significativas entre los datos de huevos de algunas especies de Crocodylidae reportados por Medem (1981, 1983), Ardila et al. (1999) y los del presente estudio, en variables como peso, largo y ancho.

Con la prueba $t$ de Student se efectuó un análisis de comparación de medias con muestras independientes de contenido de proteína en vitelo y albumen, para determinar si existían diferencias en los valores obtenidos por los métodos de Kjeldhal y de Biuret.

\section{RESULTADOS}

El peso promedio del huevo de C. intermedius fue de $121.33( \pm 20.15) \mathrm{g}, \mathrm{y}$ comparándolo con los resultados de Medem $(1981,1983)$ y de Ardila et al. (1999) no se encontraron diferencias significativas entre las muestras (ANOVA, $\mathrm{F}_{4,84}=2.89$, $\mathrm{P}>0.01)$. El peso de los componentes mayoritarios del huevo fueron: albumen 51.05 $( \pm 8.88) \mathrm{g}$, vitelo $53.33( \pm 9.9) \mathrm{g}$, cáscara $11.07 \pm(1.19) \mathrm{g}$ y cáscara con membranas $16.53( \pm 1.70) \mathrm{g}$. Con estos datos se calculó el porcentaje promedio de cáscara $(13.88 \pm 1.86)$, vitelo $(43.78 \pm 1.35)$ y albumen $(42.01 \pm 0.84)$.

Las medidas promedio de las dimensiones de los huevos fueron: diámetro mayor longitudinal $84.12 \pm 6.21 \mathrm{~mm}$, diámetro mayor transversal $51.65 \pm 1.86 \mathrm{~mm}$, longitud perimetral $228.5 \pm 14.41 \mathrm{~mm}$ y ancho perimetral $173.17 \pm 8.47 \mathrm{~mm}$.

Se estimó un índice de forma de $64.62 \pm 3.61$ para un total de 85 huevos de $C$. intermedius (datos de Medem 1981, 1983, Ardila et al. 1999).

La gravedad específica mostró valores entre $1.11-1.14$.

El grosor de la cáscara del huevo fue en promedio de $313.75 \pm 17.42 \mathrm{~mm}$ en la zona ecuatorial, $296.91 \pm 27.24 \mathrm{~mm}$ en el polo ancho y $275.91 \pm 46.80 \mathrm{~mm}$ en el polo angosto.

La medición de la fracturabilidad estableció que se requiere una fuerza de $2.34 \pm$ 0.30 Newton para fracturar un centímetro cuadrado de cáscara. La cáscara de $C$. intermedius presentó varios puntos críticos de fuerza para lograr la ruptura (Fig. 1).

El color del albumen de los huevos de $C$. intermedius es ligeramente verde, el color del vitelo amarillo pálido y su diámetro es tan grande que llega cerca de la cáscara. 
Fracturabilidad de la cáscara

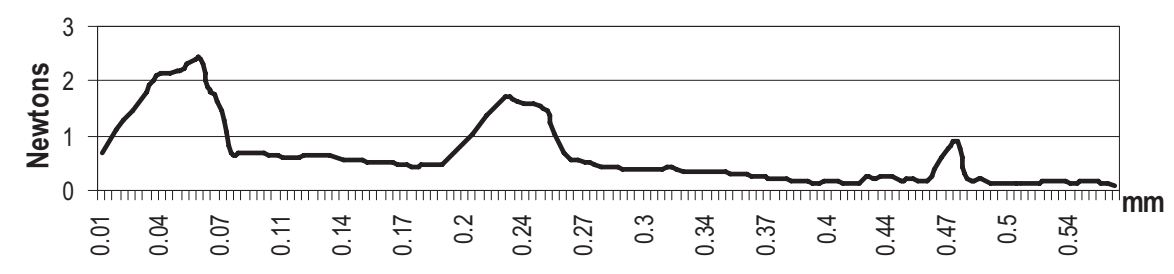

Figura 1

Comportamiento observado en el proceso de fracturabilidad de la cáscara de Crocodylus intermedius.

El color que predomina en la cáscara del huevo de C. intermedius es el blanco debido a los índices de luminosidad que presenta (L 84.22 - 88.88) y a las coordenadas cromáticas: el componente a (eje verde-rojo) presentó valores negativos entre -0.05 y -0.80 , lo que indica que domina el componente cromático verde, el componente b (eje azul-amarillo) presentó valores positivos entre 27 y 3.12 ubicándose en el área cromática amarilla.

La calidad del albumen fue en promedio de $73.70 \pm 19.53$ unidades Haugh.

La humedad en vitelo fue en promedio de $56.48 \% \pm 1.48$ y la humedad en albumen fue de $91.24 \% \pm 1.98$.

El contenido promedio de fósforo inorgánico en la cáscara del huevo fue de $0.2 \mathrm{~g}$ $(1.84 \% \pm 0.27)$ y el de calcio inorgánico $4.74 \mathrm{~g}(43 \% \pm 3.20)$. La suma de estos dos elementos representa aproximadamente la mitad del peso de la cáscara.

El porcentaje promedio de grasa total en vitelo fue de $19.30 \% \pm 4.35$.

El porcentaje promedio de proteína cruda realizado por el método Kjeldhal en vitelo fue de $18.54 \% \pm 0.93$ y en albumen fue de $6.69 \% \pm 1$. El porcentaje promedio de proteína cruda en vitelo y en albumen calculado por los métodos Kjeldhal y Biuret es significativamente diferente $(\mathrm{t}=2.65$, g.l. $=64, P=0.01$ para vitelo y albumen $)$.

\section{DISCUSIÓN}

El peso de los huevos de $C$. intermedius no difiere de los encontrados por Medem (1981) de $106.63 \mathrm{~g}$, Medem (1983) de $119.26 \mathrm{~g}$ y Ardila et al. (1999) de $123.63 \mathrm{~g}$ para esta misma especie. Este peso representa una cantidad suficiente de vitelo y albumen para mantener el desarrollo del embrión durante 90 días aproximadamente. Los huevos de $C$. intermedius son más pesados en comparación con los de otros crocodilianos, son 2.3 veces más pesados que los de Caiman crocodilus (52.81 g), 
1.83 veces más que los de Paleosuchus palpebrosus (66.17 g) y 1.73 veces más que los del híbrido P. palpebrosus x P. trigonatus (69.89 g) (Medem 1981) y son 1.78 y 1.07 veces más pesados que los de Crocodylus johnstoni $(68 \mathrm{~g})$ y C. porosus $(113 \mathrm{~g})$ respectivamente (Grahame et al. 1986).

El huevo de Crocodylus intermedius es de forma elíptica, similar a la de los huevos de otras especies de crocodilianos como $C$. crocodilus con un índice de forma de 62, P. palpebrosus 61.65, P. palpebrosus x P. trigonatus 60.73, Caiman crocodilus apaporiensis 59.83 y C. c. fuscus 58.20 (calculado a partir de Medem 1981, 1983) siendo este último el más alargado.

El porcentaje de cáscara en el huevo de Crocodylus intermedius es similar al del huevo de C. novaguineae y estos dos valores son superiores a los encontrados por Manolis et al. (1986) para C. johnstoni, C. porosus, Alligator mississippiensis y $C$. novaguineae (Cuadro 1).

\section{Cuadro 1}

Porcentaje de vitelo, albumen y cáscara de huevos de cinco especies de Crocodylia.

\begin{tabular}{lccc}
\hline Especie & \% Cáscara & \% Vitelo & \% Albumen \\
\hline C. intermedius . & $13.88 \pm 1.86$ & $43.78 \pm 1.35$ & $42.01 \pm 0.84$ \\
A. $^{\text {mississippiensis }}{ }^{1}$ & $12.0 \pm 0.3$ & $40.6 \pm 1.0$ & 46.5 \\
${\text { A. } \text { mississippiensis }^{2}}^{\text {C. johnstoni }}$ & - & - & 44 \\
C. novaeguineae $^{3}$ & $11.9 \pm 0.3$ & $46.0 \pm 0.8$ & $42.1 \pm 0.8$ \\
C. porosus & $14.0 \pm 0.9$ & - & - \\
\hline
\end{tabular}

1Dato de M.W.J. Ferguson, ${ }^{2}$ Tracy \& Snell 1985, ${ }^{3}$ Calculado de Jenkins 1975 (citados por Manolis et al. 1986).

Los huevos de $C$. intermedius poseen un proporción relativa mayor de cáscara que los huevos de A. mississippiensis, C. johnstoni, C. porosus, pero menor que los huevos de C. novaeguineae (Manolis et al. 1986). Hacia el final del desarrollo, el embrión extrae el carbonato de calcio de la cáscara para formar hueso, así el grosor de la cáscara decrece y la flexibilidad aumenta (Claessen 1979). La parte más gruesa de la cáscara en los huevos de C. intermedius se localizó en la zona ecuatorial, seguido por el polo ancho y la zona más delgada está en el polo angosto. El grosor de la cáscara se relaciona con la fracturabilidad de la misma, ya que entre más gruesa sea, más resistente a la ruptura será. El depósito de calcio en la cáscara determina que tan gruesa es ésta, si la cantidad de calcio es baja la 
cáscara será mas delgada y se romperá con mayor facilidad en la oviposición o durante los procedimientos de recolección, transporte e incubación en el caso de manipular las nidadas.

En los huevos de C. intermedius hay $0.95 \mathrm{~g}$ de albumen por cada gramo de vitelo, mientras que en $C$. johnstoni y en $C$. porosus hay $0.91 \mathrm{~g}$ y $1.07 \mathrm{~g}$ de albumen respectivamente (calculado a partir de Manolis et al. 1986), lo que indica una relación vitelo/albumen aproximada de 1/1 para estas especies de crocodilianos. El albumen es importante en la alimentación del embrión, sirve como reserva de agua y proporciona una envoltura protectora contra ataques mecánicos y químicos. El albumen de los huevos de $C$. intermedius representa aproximadamente el $42 \%$ del peso total de los mismos, equivalente al encontrado en los huevos de C. johnstoni (Manolis et al. 1986) y cercano a los valores determinados en los huevos de $C$. porosus, y A. mississippiensis. Si se toma como proporción sólo la combinación del peso de vitelo y albumen, el vitelo representa el 51.1\% del contenido del huevo en C. intermedius, similar al de otros crocodilianos como C. johnstoni $(52.2 \%), C$. porosus (48.1\%) y A. mississippiensis (46.6\%). En C. intermedius y C. johnstoni el vitelo es el mayor componente del huevo, mientras que en especies como $A$. mississippiensis y C. porosus (Manolis et al. 1986) el porcentaje de vitelo es menor (Cuadro 1).

En los huevos de C. intermedius el agua es el componente mayoritario del albumen y la porción en materia seca restante es proteína, además de cantidades no discernibles de grasa, composición similar a la de los huevos de C. johnstoni (Manolis et al. 1986).

El color del albumen, el vitelo y el diámetro del mismo en los huevos de $C$. intermedius coincide con lo observado en otros crocodilianos (Goin \& Goin 1971). El porcentaje inicial de humedad en el albumen de los huevos de crocodilianos facilita que del albumen se transfiera agua y proteína para formar el fluido subembrionario, de modo que el contenido de agua en el albumen disminuye con el tiempo (Gans \& Huey 1982). La manera en que se reduce el contenido inicial de humedad en el albumen de huevos fértiles de C. intermedius en cada etapa de desarrollo del embrionario es un aspecto aun no investigado.

El contenido de fósforo inorgánico en el organismo debe estar en una proporción óptima en relación con el calcio ya que una dieta rica en fósforo puede producir deficiencia de calcio. En la etapa reproductiva de las hembras de crocodilianos las variaciones radicales en el metabolismo del calcio interfieren con la formación normal de la cáscara del huevo (Lance 1986). El porcentaje de calcio inorgánico en la cáscara del huevo es un valor determinante ya que el embrión usa el calcio en la osificación del esqueleto y del 63 al 70\% de éste es obtenido de la cáscara. Hacia el final del desarrollo el embrión extrae carbonato de calcio de la cáscara para formar hueso, así el grosor de la cáscara decrece y la flexibilidad aumenta (Claessen 1979). 
Aproximadamente $4.74 \mathrm{~g}$ de calcio son depositados en la cáscara de un huevo de $C$. intermedius, por lo tanto una hembra que oviposite 70 huevos requerirá $331.8 \mathrm{~g}$ de calcio que no pueden ser tomados de la dieta y de llegar a hacerlo no se movilizaría lo suficientemente rápido para que del intestino delgado llegue al oviducto, por lo que el calcio se debe movilizar de los huesos (Lance 1986). Desde el punto de vista del manejo de $C$. intermedius en cautiverio, conociendo estos requerimientos se pueden formular dietas con suficiente contenido de calcio previo a la postura para que el calcio necesario se deposite a nivel óseo, evitando pérdidas de huevos por baja calidad de la cáscara.

Se decidió tomar como valores de referencia los datos obtenidos por el método de Kjeldhal para los valores de proteína, ya qué este arrojó valores más homogéneos y acordes con los conocidos en otras especies de crocodilianos (Manolis et al. 1986). El porcentaje de proteína cruda en vitelo debe garantizar al embrión un suministro suficiente de proteína ya que ésta porta vitaminas, oxígeno y bióxido de carbono, además de tener propiedades estructurales, cinéticas, catalíticas y de señalización. El porcentaje de proteína en peso seco del vitelo en huevos de $C$. intermedius fue de $42.53 \%$, mientras en C. johnstoni fue de $45.82 \%$ (Manolis et al. 1986). El porcentaje de proteína cruda en albumen es aproximadamente el $76.37 \%$ en peso seco en $C$. intermedius valor más bajo si se compara con C. johnstoni (86\%) (Manolis et al. 1986).

Alrededor del $44.37 \%$ en peso seco del vitelo de $C$. intermedius está representado por grasa, mientras que en $C$. johnstoni este valor es cercano al 47\% (Manolis et al. 1986) (Cuadro 2). La grasa es el componente mayoritario de los sólidos del vitelo, sirve como fuente principal de alimento para el embrión debido a que es una importante fuente energética por contener más energía por unidad de peso, de la cual el embrión puede extraer el 95\% mientras que sólo extrae el $77 \%$ de la energía potencial de las proteínas.

\section{Cuadro 2}

Contenido de agua, proteína y grasa de los huevos de tres especies de Crocodylidae al momento de la postura.

\begin{tabular}{|c|c|c|c|c|c|c|}
\hline \multirow{2}{*}{ Especie } & \multicolumn{3}{|c|}{ Albumen } & \multicolumn{3}{|c|}{ Vitelo } \\
\hline & $\%$ agua & \% proteína & $\%$ grasa & $\%$ agua & \% proteína & $\%$ grasa \\
\hline C. intermedius & 91.24 & 6.69 & - & 56.48 & 18.51 & 19.31 \\
\hline C. porosus ${ }^{1}$ & 96.4 & - & - & 56.4 & - & - \\
\hline C. johnstoni ${ }^{1}$ & 95.0 & 4.3 & - & 58.1 & 19.2 & 19.7 \\
\hline
\end{tabular}

Fuente: ${ }^{1}$ Manolis et al. (1986). 


\section{AGRADECIMIENTOS}

Los autores agradecen a María Cristina Ardila, directora de la Estación de Biología Tropical Roberto Franco de la Universidad Nacional de Colombia por la donación del material biológico sobre el cual se realizó el presente estudio. A los docentes Alirio Fajardo por sus reflexiones y orientaciones, Jairo Granados por guiarnos en el desarrollo de los análisis, Luis Alfonso Bocanegra por sus acertados comentarios en la corrección del manuscrito. Al doctor Cesar Julio Zabala por su motivación, a la Universidad de Cundinamarca donde se realizó la mayor parte de este trabajo. A Alberto Suárez y Eliana Torres por su colaboración en el trabajo de laboratorio. A Hugo Humberto Ballesteros investigador del Programa Nacional de Recursos Genéticos de Corpoica, sede Tibaitatá, por su asistencia en la determinación del color y fracturabilidad de la cáscara. A los docentes Cesar Tinoco y Mary Dupont por sus oportunas aclaraciones metodológicas. A cada una de las personas que colaboraron con su tiempo, paciencia, conocimiento y voluntad. A todas ellas queremos agradecer sinceramente el haber prestado su atención a este trabajo.

\section{LITERATURA CITADA}

Ardila-R., M.C, S.L. Barahona-B., O.P. Bonilla-C. \& D.R. Cárdenas-R. 1999. Aportes al conocimiento de la reproducción, embriología y manejo de Crocodylus intermedius en la Estación de Biología Tropical "Roberto Franco" de Villavicencio. Rev Acad. Colomb. Cienc., 23 (suplemento especial): 417-424.

Association of Official Analytical Chemists. (A.O.A.C). 1984. Eggs. Pp. 320-329. In: (ed) S. Williams. Official methods of analysis of the Association of official Analytical Chemists. Centennial, Virginia.

Claessen, H. 1979. Reproduction eggs: factors associated with incubation and hatching and suggestion for laboratory rearing. J. Herpetol .13 (4): 472-475.

Comisión Internationale de l'Eclariae (CIE). 1986. Colorimetry. 2 ed. CIE Publications. $\left(\mathrm{N}^{\circ} 15.2\right)$. Viena.

Comité Técnico AEN/CTN 77 Medio Ambiente. 2002. Calidad del agua. Determinación de la dureza del agua. Método complexométrico con AEDT. UNE 77040. AENOR, Madrid.

Gans, C. \& R. Huey. 1982. The physiological ecology of the reptilian egg and embryos: Types of eggs. Pp. 528-529. In: Biology of the reptilia. Vol 16. Academic Press. New York.

Goin, C \& O. Goin. 1971. Introduction to herpetology. Second Edition. W.H. Freeman and company. San Francisco.

González, L. J. 1986. Parámetros fisicoquímicos de la cáscara de huevo: Importancia. Pp. 28-50. In: González, L. J. Parámetros fisicoquímicos de la cáscara del huevo. Importancia.: Universidad Nacional de Colombia Seccional Medellín. Medellín.

Grahame, W. \& C. Manolis. 1986. Methods for retrieving crocodilian embryos. Pp. 423426. In: Grahame Weeb et al. (eds). Wildlife management: crocodiles and alligators. Surrey Beatty \& Sons Pty Limited. Winnellie. 
Grahame, W.; W. Webb; P. Whiteheat \& W. Manolis. 1986. Crocodilian management in the northern territory of Australia. Pp 113. In: Grahame Weeb et al. (eds). Wildlife management: crocodiles and alligators. Surrey Beatty \& Sons Pty Limited. Winnellie.

Instituto Mexicano de Normalización y Certificación, A.C. 2004. Apéndices normativos: métodos de medición para determinar el grado de clasificación del huevo. Pp 25-26. In: Productos avícolas -huevo fresco de gallina- especificaciones y métodos de prueba. (NMX-FF-079-SCFI-2004). Secretaria de Economía. Ciudad de México.

Lance, V. 1986. Hormonal control of reproduction in crocodilians. Pp 409-415. In: Grahame Weeb et al. (eds). Wildlife management: crocodiles and alligators. Surrey Beatty \& Sons Pty Limited. Winnellie.

Manolis, C. W. Grahame \& K. Dempsey. 1986. Crocodile egg chemistry. In: Grahame Weeb et al. (eds). Wildlife management: crocodiles and alligators. Surrey Beatty \& Sons Pty Limited. Winnellie.

Medem, F. 1981. Los Crocodylia de Sur América, Vol. I: Los Crocodylia de Colombia. COLCIENCIAS-Universidad Nacional de Colombia. Bogotá. - 1983. Los Crocodylia de Sur América Vol. II. COLCIENCIAS-Universidad Nacional de Colombia. Bogotá.

Ramírez, J. 1999. Técnicas de recolección y control de calidad de huevos de tortugas y cocodrilos In: Ramírez, J. Zoodivulgación. GM Editores, Villavicencio.

Rodríguez, M. \& J. Ramírez. 2002. Crocodylus intermedius. Pp 45-48. In: Castaño-Mora, O. V. Libro rojo de reptiles de Colombia. Libros rojos de especies amenazadas de Colombia. Instituto de Ciencias Naturales-Universidad Nacional de Colombia, Ministerio del Medio Ambiente, Conservación Internacional-Colombia. Bogotá.

Rueda-A, J. V. 1999. Anfibios y reptiles amenazados de extinción en Colombia. Rev Acad. Colomb. Cienc. 23 (suplemento especial): 475-498.

Thorbjarnarson, J. B. \& G. Hernández. 1993. Reproductive ecology of the Orinoco crocodile (Crocodylus intermedius) in Venezuela. I. Nesting ecology end egg and clutch relationships. J. Herpetol. 27 (4): 363-370.

UICN. 2001. Categorías y Criterios de la Lista Roja de la UICN: Versión 3.1. Comisión de Supervivencia de Especies de la UICN. UICN, Gland, Suiza y Cambridge, Reino Unido. ii $+33 \mathrm{pp}$.

Universidad de Oviedo. 2005. Determinación de fosfatos en fertilizantes. [online]. Universidad de Oviedo. Facultad de Química. Oviedo. 2003. [consultado 23 de abril 2005]. <www.uniovi.es/QF Analitical/trans/quimforest/práctica4.pdf>.

Recibido: 15 de febrero de 2006

Aceptado: 20 de abril de 2007 